\title{
Effects of prior knowledge on learning from different compositions of representations in a mobile learning environment
}

\author{
Tzu-Chien Liu ${ }^{\mathrm{a}, *}$, Yi-Chun Lin ${ }^{\mathrm{a}}$, Fred Paas ${ }^{\mathrm{b}, \mathrm{c}}$ \\ ${ }^{a}$ National Central University, Graduate Institute of Learning and Instruction, No.300, Jhongda Rd., Jhongli City, Taoyuan County 32001, Taiwan \\ ${ }^{\mathrm{b}}$ Erasmus University Rotterdam, Institute of Psychology, Burgemeester Oudlaan 50, 3062 PA Rotterdam, Netherlands \\ ${ }^{\mathrm{c}}$ University of Wollongong, Faculty of Education, Wollongong, NSW 2522, Australia
}

\section{A R T I C L E I N F O}

\section{Article history:}

Received 24 June 2013

Received in revised form

9 September 2013

Accepted 30 October 2013

\section{Keywords:}

Applications in subject areas

Elementary education

Pedagogical issues

Teaching/learning strategies

\begin{abstract}
A B S T R A C T
Two experiments examined the effects of prior knowledge on learning from different compositions of multiple representations in a mobile learning environment on plant leaf morphology for primary school students. Experiment 1 compared the learning effects of a mobile learning environment presenting text and photos of plants on a tablet PC, either in combination with or without real plants in the physical environment. Results indicated that there were no interactions between prior knowledge and experimental condition. Students who learned with tablet PCs only outperformed students who additionally learned with real plants on a comprehension and an application test. In addition, high prior knowledge students outperformed low prior knowledge students on both tests. To investigate whether these effects were caused by the specific characteristics of the combination of photos of real plants and real plants, Experiment 2 compared the differential effects of prior knowledge on learning with the combination of texts, photos and real plants to a combination in which the photos were replaced by schematic hand drawings. Results indicated that both low and high prior knowledge students, who learned with the combination of texts, schematic hand drawings and real plants performed better on a comprehension and an application test. High prior knowledge students performed better on both tests. It is concluded that the number and type of representations used is critical for the effectiveness of mobile learning environments.
\end{abstract}

(c) 2013 Elsevier Ltd. All rights reserved.

\section{Introduction}

Mobile device supported learning in physical environments has been successfully applied in different fields (Liu, Peng, Wu, \& Lin, 2009; Sung, Chang, Hou, \& Chen, 2010). However, the cognitive processes underlying mobile device supported learning have seldom been explored (Liu, Lin, Tsai, \& Paas, 2012). When learning with a mobile device in physical environments, students are typically provided with multiple representations, such as texts and photos embedded in a mobile device and authentic objects in physical environments (e.g., birds, plants) (Chen, Kao, \& Sheu, 2003; Lai, Yang, Chen, Ho, \& Chan, 2007; Liu et al., 2009). As indicated in the DeFT (Design, Functions, Tasks) framework for learning with multiple external representations (Ainsworth, 2006), each of the individual representations can complement one another to enhance the understanding of a complicated single representation. Although this complementary function in the use of multiple representations may have positive effects on learning by creating a rich learning environment, it may also negatively affect learning by imposing a too high extraneous cognitive load (Liu, Lin, \& Paas, 2013). Therefore, it is critical to investigate instructional guidelines that may avoid the negative effects and stimulate the positive effects of mobile device supported learning in physical environments.

The interaction of learners with authentic objects, which constitute an important source of information in mobile device supported learning in physical environments, has been found to positively affect student involvement and learning performance (Brown, Collins, \& Duguid, 1989; Heinich, Molenda, Russell, \& Smaldino, 2001). In addition, Paas and Sweller (2012) have used Geary's (2007, 2008, 2012) evolutionary educational psychology approach to argue that interacting with authentic objects, such as plants, can be considered as a form of effortless learning that we have evolved to acquire (i.e., biologically primary knowledge), and which can be used to facilitate more

\footnotetext{
* Corresponding author. Tel.: +8863 4227151x33860; fax: +886 34273371.

E-mail addresses: 1tc@cc.ncu.edu.tw (T.-C. Liu), 961407001@cc.ncu.edu.tw (Y.-C. Lin), paas@fsw.eur.nl (F. Paas).
} 
effortful forms of learning that we have not evolved to acquire (biologically secondary knowledge), such as memorizing the different verbal definitions used in plant leaf morphology.

Cognitive load theory is concerned with optimizing learning by designing instructional methods that efficiently use people's limited working memory resources (CLT: Chandler \& Sweller, 1991; Paas, Renkl, \& Sweller, 2003; Paas \& Van Merriënboer, 1994; Sweller, Ayres, \& Kalyuga, 2011; Sweller, Van Merriënboer, \& Paas, 1998). There are three main types of cognitive load. Intrinsic cognitive load is determined by the complexity of the content that has to be learned, which in turn depends on element interactivity as well as on learners' prior knowledge. Extraneous cognitive load refers to the working memory resources required to process unnecessary information due to poor instructional design. Germane cognitive load is associated with the presentation of information or the design of an activity to foster learning (Sweller et al., 1998).

By comparing different compositions of learning sources in mobile device supported learning in a natural environment, Liu et al. (2012) investigated redundancy and split-attention effects that have been shown to negatively affect students' learning. According to cognitive load theory (Chandler \& Sweller, 1991; Sweller et al., 1998), a redundancy effect may occur when learning materials consist of multiple sources of information that are self-contained and can be used without reference to each other, and when learning materials contain a source of information that overlaps with knowledge that is already available in the learner's long term memory (e.g., Cerpa, Chandler, \& Sweller, 1996; Kalyuga, Chandler, \& Sweller, 1999; Rasch \& Schnotz, 2009). The use of redundant sources of information, such as simultaneous presentation of the same text in spoken and written format, has a negative effect on learning, because it imposes an unnecessary working memory load (e.g., Kalyuga et al., 1999; Mayer \& Johnson, 2008). In Liu et al.'s (2012) study the finding that students who worked with texts and photos of real plants and those who worked with texts and real plants learned more than students who worked with texts, photos of real plants, and real plants, was indicative for a redundancy effect.

A split-attention effect may occur if learners have to split their attention between multiple sources of information that must be mentally integrated before the learning task can be understood (e.g., Ayres \& Sweller, 2005; Bobis, Sweller, \& Cooper, 1993; Kalyuga et al., 1999; Liu \& Lin, 2011). Liu et al. (2012) could not confirm their hypothesis that the split-attention effects would be more pronounced when the learners had to mentally integrate the texts that were presented on the mobile device with the real plants that were available in physical environments than when they had to mentally integrate the texts and photos of the real plants that were both presented on the mobile device.

It is important to note that the impact of media compositions on learning, both in terms of redundancy effects (e.g., Kalyuga, 2007; Rey \& Buchwald, 2011) and split-attention effects (e.g., Kalyuga, Chandler, \& Sweller, 1998, 2000), may differ as a function of the student's level of prior knowledge. According to the expertise reversal effect (Kalyuga, Ayres, Chandler, \& Sweller, 2003; Sweller et al., 2011), instructional techniques that are effective at a certain level of prior knowledge, such as physical integration of two or more sources of information that are not intelligible in isolation can lose their effectiveness and even have negative consequences when used at another level of prior knowledge. We expected that an expertise reversal effect would also materialize with different compositions of representations in mobile learning environments. Although realistic photographs are frequently used in mobile learning environments, previous research has shown that it is a less effective visual illustration format for complementing verbal representations than simple line drawings (Dwyer, 1967; cited in Dwyer, 1972). In addition, in the study of Dwyer (1972) (cited in Dwyer, 1972), low prior knowledge students were found to spend more learning time and achieve lower test performance than high prior knowledge students when using realistic photographic presentations complementing oral presentation. Based on the findings of Dwyer (1972), it could be argued that in contrast to more advanced students, low prior knowledge students may not profit from the high level of realism and detail in realistic representations (e.g., real plants used by Liu et al., 2012), because they are not able to distinguish between essential and non-essential elements of the representations. Consequently, they learn more from schematic representations, which typically present only the essential features (e.g., Moreno, Ozogul, \& Reisslein, 2011). In addition, whereas low prior knowledge students tend to focus on superficial aspects of a representation (e.g., Cook, Wiebe, \& Carter, 2008), more advanced students can see beyond surface features and are able to identify structural aspects of a representation (e.g., Chi, Glaser, \& Farr, 1988; Chi, Glaser, \& Rees, 1982). With regard to the materials used in the present study on plant leaf morphology (see also Liu et al., 2012), the superficial aspects that can easily be identified by novices in photos or real plants are, for example, the leaf color (e.g., green, red or yellow) and the rough leaf shape (e.g., circle or elliptic). The structural aspects that novices cannot be easy to identify in the photos or real plants are, for example, the type of venation and margin of the plant leafs.

Due to these differences between low and high prior knowledge students, an interesting phenomenon may emerge in learning environments that combine authentic objects with photos of those objects. It can be assumed that low prior knowledge students who tend to focus on superficial similarities may not be able to recognize the structural differences between a real plant and a photo of that plant, and consequently not be able to profit from the complementary function of the multiple external representations (Ainsworth, 2006). So, for the low prior knowledge students the multiple representations would be intelligible in isolation with one source being redundant. The results may be different for the high prior knowledge students because they are able to make structural comparisons and recognize structural differences between the real plants and photos of those plants. Consequently, they may profit from the complementary function of the multiple representations (Ainsworth, 2006). However, learners would need to split their attention between, and mentally integrate the spatially separated representations for this potential positive effect to materialize.

In this study, two experiments were conducted in a mobile learning environment on leaf morphology of plants, which is an important learning topic in primary schools' nature science curriculum in Taiwan. The first experiment compared the learning effects of a mobile learning environment presenting text and photos of plants on a tablet PC, either in combination with (TPO) or without real plants (TP) in the physical environment. Experiment 2 compared the differential effects of prior knowledge on learning in a TPO condition to a condition in which the photos were replaced by schematic hand drawings (THO).

Whereas the study of Liu et al. (2012) focused on split-attention and redundancy effects, the effect of prior knowledge on both effects was not explored. Therefore, the main question that will be addressed in the first experiment of this study is whether the level of prior knowledge of students affects their ability to profit from the combination of text, photos of plants, and real plants. It was expected that low prior knowledge students' learning would be negatively affected by this combination of information sources resulting from a redundancy effect. However, for high prior knowledge students a split-attention effect was expected, because they are able to identify the structural differences between the photos of the authentic objects and the authentic objects. To profit from the rich information represented by the authentic objects and photos of the authentic objects, they would need to mentally integrate these spatially separated information sources, 
which creates a split-attention effect. However, it is not possible to formulate a specific hypothesis for the effect of multiple representations on learning of high prior knowledge students, because their performance will be determined by the sum of the positive complementary effect and the negative split-attention effect of the multiple spatially separated representations.

Whereas the first experiment was based on the redundancy effect that learning of low prior knowledge students can be improved by eliminating the authentic objects from the mobile device supported learning environment, the second experiment investigated an alternative approach. This approach was based on the assumption of the complementary function of multiple representations, which holds that the information conveyed by different representations can complement each other to enhance the understanding of a complicated single representation (Ainsworth, 2006), but only if students are able to perceive the differences between the representations. As suggested by Liu et al. (2012) an instructional strategy that might enable students to perceive the differences between different representations, and prevent them from redundancy effects is to decrease the superficial similarity between the photos and the authentic objects, for example by using schematic hand drawings instead of pictures. The main question that will be addressed in the second experiment of this study is whether the use of a schematic hand drawing of a plant would enable low prior knowledge students to profit more from the complementary information conveyed by hand drawings and real plants.

A hand drawing is a kind of pictorial representation that is widely used in text books. In contrast to a photo a hand drawing can be used to emphasize important aspects to be learnt and eliminate irrelevant details that are normally present in authentic objects or photos of those objects. Therefore, hand drawings and authentic objects are predicted to complement each other in mobile device supported learning in the physical environment. For example, when learning the features of plant leaf with the assistance of a mobile device, students can first read the text that introduces the target features of the plant leaf, then observe the corresponding target features in the hand drawing embedded on the mobile device, and then observe the leaf on a real plant. Whereas, the information conveyed by a hand drawing could benefit students to comprehend the features of the plant leaf, the information conveyed by the real plant could benefit students to transfer what they have learnt in identifying the features in new plants.

\section{Experiment 1}

The aim of Experiment 1 was to examine whether redundancy and split-attention effects would emerge as a function of different combinations of multiple representations for low and high prior knowledge students, respectively.

Low prior knowledge students were expected to experience a redundancy effect in the condition with text, photos of plants and real plants, because they do not yet have a cognitive schema that allows them to identify the structural differences between the photo of a plant and the real plant, thereby making both representations intelligible in isolation and one representation being redundant. Therefore, it was hypothesized that low prior knowledge students who would learn with the text and photos on a mobile device would need less time to learn and achieve better performance on a comprehension and an application test than low prior knowledge students who would learn with text and photos of plants on a mobile device and real plants in the physical environment (Hypothesis 1a).

In contrast, a split-attention effect was predicted for high prior knowledge students when dealing with the spatially separated information sources. High prior knowledge students were expected to have a cognitive schema that allows them to make structural comparisons and identify structural differences between the photo of a plant and the real plant, thereby enabling them to profit from the complementary function of both representations. Therefore, it was hypothesized that high prior knowledge students would achieve better performance on a comprehension and an application test in a learning condition with text and photos of plants on a mobile device and real plants in the physical environment than in a learning condition with only text and photos on a mobile device (Hypothesis 1b). Although it was expected that the high prior knowledge students could profit from the possibility to interact with real plants, this benefit would be inhibited by a split-attention effect resulting from mentally integrating spatially separated information from photos of the plants and the real plants which are unintelligible in isolation for them. With regard to students' prior knowledge, it was hypothesized that students with high prior knowledge would generally show better learning performance, and need less learning time than students with lower prior knowledge in all learning conditions (Hypothesis 2).

\subsection{Method}

\subsubsection{Participants}

Seventy-eight fifth-grade students (43 boys and 35 girls) of a primary school in Taiwan participated in this study. The mean age of the students was around 11 years. Because we could not collect the personal data, such as the children's age, from the school during the experiment, these data were collected from the web site of the Ministry of Education (Taiwan). All participants had been taught the essential concepts about leaf morphology of plants by the same teacher 18 months before the start of the experiment.

In order to obtain two groups of learners with distinctively different levels of prior knowledge, an extreme group design was adopted. One week before the formal experiment, the participants were administered a prior knowledge test that was composed of ten multiplechoice items. Participants were divided into two groups (high prior knowledge and low prior knowledge) according to their scores on the prior knowledge test. The highest score for the test was 10 points. Six points was used as a cutoff point and the twelve participants who got 6 points were not invited to participate in the formal experiment. Thirty participants (about $40 \%$ of participants) who had a pre-test score lower than 6 points were selected as the low prior knowledge group $(M=4.30, S D=1.09$ ). Thirty-six participants (about $46 \%$ of participants) who had a pre-test score higher than 6 points were selected as high prior knowledge students $(M=7.80, S D=0.81)$, thirty of them were randomly selected from this group to create an equally sized high prior knowledge group.

\subsubsection{Design}

A 2 (composition of representations: text and photo vs. text, photo, and real plant) $\times 2$ (prior knowledge: high vs. low) between-subjects design was used to address the hypotheses in this study. The low and high prior knowledge participants were randomly assigned to the two conditions with different compositions of multiple representations, resulting in 15 participants in each of the four experimental conditions; students with high prior knowledge learning with text and photos of plants, students with high prior knowledge learning with text, photos 
of plants, and real plants, students with lower prior knowledge learning with text and photos of plants, students with lower prior knowledge learning with text, photos of plants, and real plants.

\subsubsection{Materials}

The learning materials in this study focused on plant leaf morphology, which is an important learning topic in the primary schools' nature science course in Taiwan. The learning materials (TP and TPO) were adapted from the learning materials used in the study of Liu et al. (2012). There were two differences of the learning materials used in the current study and in the study of Liu et al. (2012). Firstly, in this study the basic knowledge of leaf features was introduced before the formal learning phase, instead of during the formal learning phase. Secondly, the photos of the plants used in the study of Liu et al. (2012) were replaced by new ones in this study. The learning materials were revised in collaboration with one primary school teacher, who held a Master's degree in nature science education.

Learning materials for introducing the basic knowledge of leaf features (including the names of the different types of venation, margin, and phyllotaxy) consisted of five PowerPoint slides.

Learning materials for introducing the three features of leaves (including venation, margin, and phyllotaxy of the plant leaf) were presented either by texts and photos of plants that were embedded in a tablet PC (i.e., the TP condition), or by texts and photos of plants that were embedded in a tablet PC, and the authentic objects (O) (i.e., real plants) that were available in the physical environment (i.e., TPO condition). The learning materials consisted of 26 screens. The first screen introduced each learning condition with the specific version of the learning materials. The next 24 screens, six plants placed in a line were used as examples to introduce venation, margin, and phyllotaxy as the three features of leaves. Fig. 1 depicts the differences between the experimental conditions using the fourth screen of the experiment as an example. This screen presented the green maple plant to introduce the decussate phyllotaxy. Note that in all conditions, text and

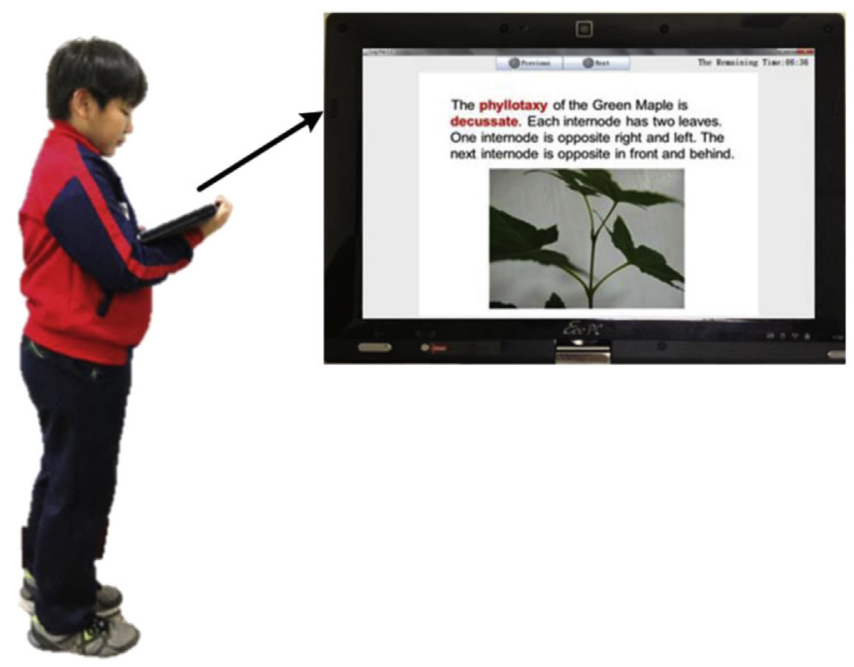

(a)

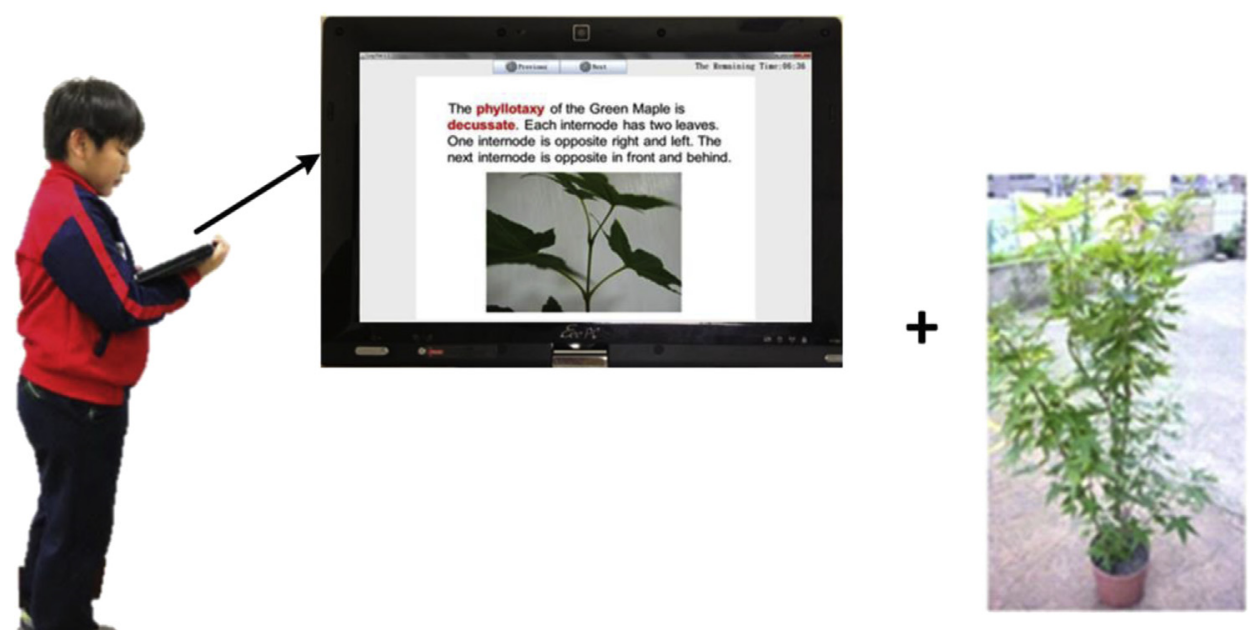

(b)

Fig. 1. The learning materials of the TP (a) and TPO (b) conditions. 
photos were presented on a mobile device (i.e., tablet pc). The final screen was used to thank the students for their participation. All texts were presented in Chinese language.

\subsubsection{Apparatus}

The equipment used in this experiment was an i-pad like tablet pc with a 10-inch monitor. The instructional software that was used on the tablet pc was developed in JAVA. The instructional software allowed students to learn at their own pace and recording of students' learning time in a database.

\subsubsection{Measures}

A prior knowledge test, consisting of ten multiple-choice questions was used to determine students' prior knowledge about plant leaf features. The highest and lowest possible score for the test was 10 points and 0 point, respectively (each correct answer scored 1 , and each wrong answer scored 0 ). The internal consistency reliability coefficient (KR-20) of the test was found to be 0.81 .

A comprehension test was used to measure participants' comprehension performance after learning. The test consisted of two types of tasks, a drawing and an assembling task. The drawing task consisted of 8 items, in which the students were asked to draw the four types of venation and four types of margins. Because the leaf phyllotaxy was difficult to depict in a drawing, an assembling task consisting of five items was used to examine participants' knowledge of leaf phyllotaxy (Liu et al., 2012, 2013). In the assembling task the students were required to present the five types of phyllotaxy by placing the correct artificial leaves on five artificial stems.

An experienced nature science teacher scored the students' performance on the two tasks. The highest and lowest possible score for the two tasks was 13 points and 0 point (each correct answer scored 1, and each wrong answer scored 0 ) and this score was used as the index of the students' comprehension performance in this experiment. The internal consistency reliability coefficient (KR-20) of the test was 0.75 .

An application test composed of 12 multiple-choice questions with 4 choices and 6 multiple-choice questions with 5 choices was used to examine whether the participants were able to apply what they had learnt in a new situation. Students were asked to identify the features of the leaves of six plants that differed from the plants used in the learning phase. The answers had to be written down on a paper based sheet. The highest and lowest possible score on this task was 18 points and 0 point (each correct answer scored 1, and each wrong answer scored 0). The internal consistency coefficient (KR-20) of the test was 0.72. Fig. 3 depicts the comprehension and application tests of the research.

\subsubsection{Procedure}

Before the formal experiment, a pilot study was conducted to determine maximum learning time limits, testing time limits and students' understanding of the learning materials. Thirty-one fifth-grade students (Mean age 11 years; 18 boys and 13 girls) that were randomly selected from another class of the same school participated in this study.

The experiment consisted of three consecutive phases, i.e. an introductory phase, a learning phase, and a test phase. The students participated individually in all phases.

2.1.6.1. Introductory phase. In this phase the students had 3 min to read an introductory text about basic knowledge of plant leaf features that was presented on the tablet PC.

2.1.6.2. Learning phase. Individual students in the different conditions were given the corresponding version of the learning materials in this phase. Before the individual students started learning, the experimenter asked the students in the TP condition to learn the leaf features by reading the text and photos embedded in the tablet PC, and the students in the TPO condition by reading the text and photos embedded in the tablet PC and observe the corresponding real plants that were placed in a line in a botanical garden-like learning environment. Students could control the presentation of the instructional material by an electronic pen. The time limit, which was determined in a pilot study, was $7 \mathrm{~min}$. The students could stop at any time within the $7 \mathrm{~min}$ and each student's learning time and learning pace were recorded on the tablet PC.

2.1.6.3. Test phase. Immediately after completing the learning phase, participants had 8 min to complete the comprehension test, which consisted of the drawing and assembling task, and 6 min to complete the application test.

\subsection{Results and discussion}

The data of the learning phase were analyzed with 2 (composition of representations: TP vs. TPO) $\times 2$ (prior knowledge: High vs. Low) univariate analyses of variance (ANOVAs) with learning time as dependent variable, and multivariate analyses of variance (MANOVAs) with comprehension and application test scores as dependent variables. Statistical significance for all tests was set at $p<0.05$. The data of the learning and test phases are presented in Table 1.

\subsubsection{Learning time}

For the dependent variable learning time, the ANOVA revealed no main effect of composition of representations, $F(1,56)<1, n s$. The main effect of prior knowledge was significant, $F(1,56)=11.41, p<0.01$, MSE $=1.70, \eta_{p}^{2}=0.17$. Participants with high prior knowledge spent significantly less time on learning than the participants with low prior knowledge. The interaction between composition of representations and prior knowledge failed to reach statistical significance, $F(1,56)<1, n s$.

\subsubsection{Comprehension and application performance}

For the dependent variables comprehension and application test performance the MANOVA revealed a main effect of composition of representations, Wilks's $\lambda=0.78, F(2,55)=7.83, p<0.01, \eta_{p}^{2}=0.22$, with participants in the TP condition performing better than the participants in the TPO condition. In addition, a significant main effect of the prior knowledge was found, Wilks's $\lambda=0.48, F(2,55)=29.42$, 


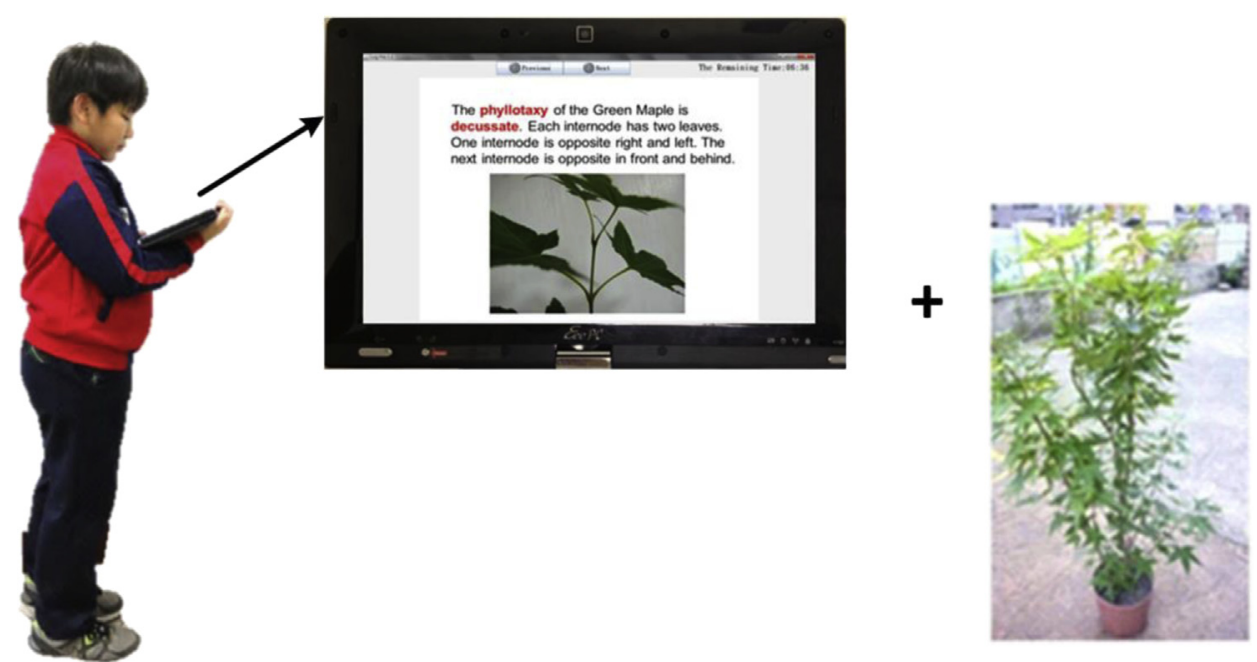

(a)
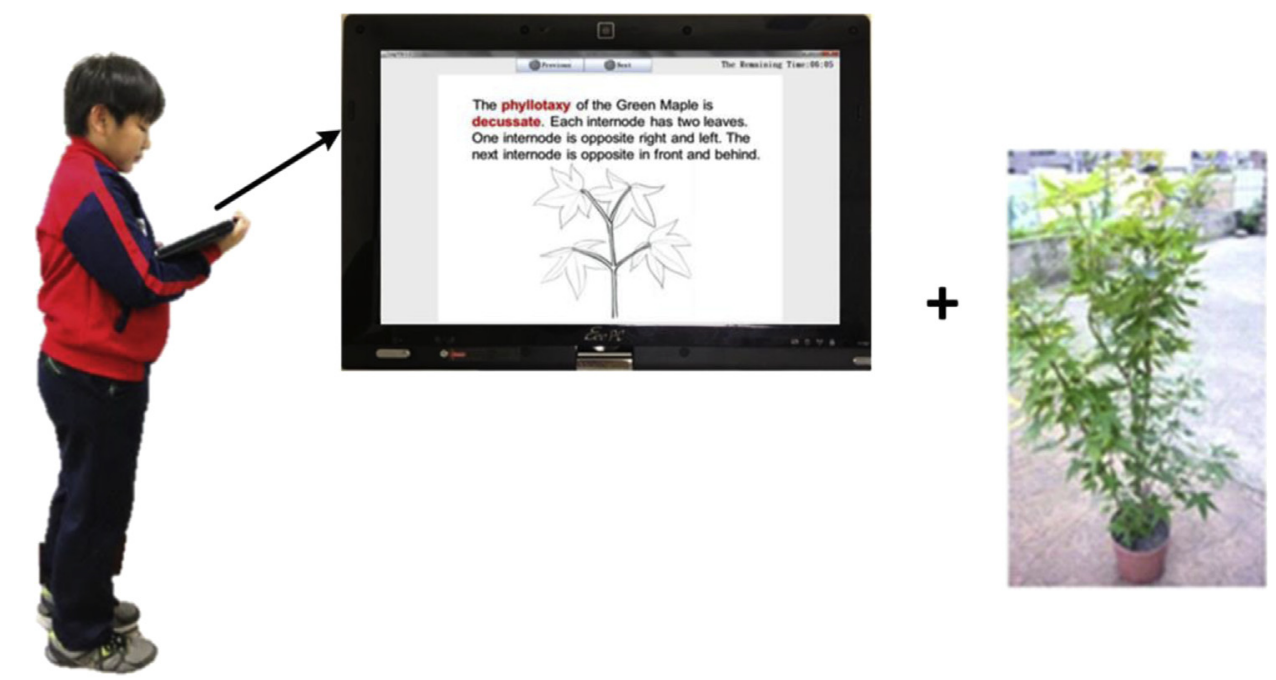

(b)

Fig. 2. The learning materials of the TPO (a) and THO (b) conditions.

$p<0.001, \eta_{p}^{2}=0.52$, with high prior knowledge participants performing better than the low prior knowledge students. The interaction between composition of representations and prior knowledge was not significant, Wilks's $\lambda=0.92 ; F(2,55)=2.28, n s\left(p=0.112, \eta_{p}^{2}=0.08\right.$, observed power $=0.45$ ).

The follow-up univariate ANOVAs that were computed for comprehension performance with the Bonferroni correction set at alpha level $<0.05 / 2=0.025$, revealed significant main effects of composition of representations, $F(1,56)=13.52, \mathrm{MSE}=4.74, p<0.01, \eta_{p}^{2}=0.19$, and prior knowledge, $F(1,56)=24.81$, MSE $=4.74, p<0.001, \eta_{p}^{2}=0.31$. The main effects indicated that participants in the TP condition performed better than the participants in the TPO condition, and that the high prior knowledge students performed better than the low prior knowledge students. The interaction between composition of representations and prior knowledge failed to reach statistical significance, $F(1,56)=4.56$, $n s$ (MSE $=4.74, p=0.037 ; \eta_{p}^{2}=0.08$, observed power $=0.56$ ).

With regard to application performance, the main effect of composition of representations was significant, $F(1,56)=6.31, \mathrm{MSE}=4.89$, $p<0.025, \eta_{p}^{2}=0.10$, with participants in the TP condition performing better than the participants in the TPO condition. The main effect of prior knowledge was also significant, $F(1,56)=49.95$, MSE $=4.89 ; p<0.001, \eta_{p}^{2}=0.47$, indicating that participants with high prior knowledge performed significantly better than the participants with low prior knowledge. The interaction between composition of representations and prior knowledge failed to reach statistical significance, $F(1,56)<1, n s$.

For two of the three dependent variables (i.e., comprehension and application performance) significant main effects were found for the independent factors composition of representations and prior knowledge. The absence of an interaction between composition of representations and prior knowledge indicated that these results were not differentially affected by one of these factors. The results indicated that participants who worked with text and photos of real plants on the mobile device achieved higher performance on the comprehension and application tests than participants, who worked with text and photos on the mobile device and real plants in the physical environment. 


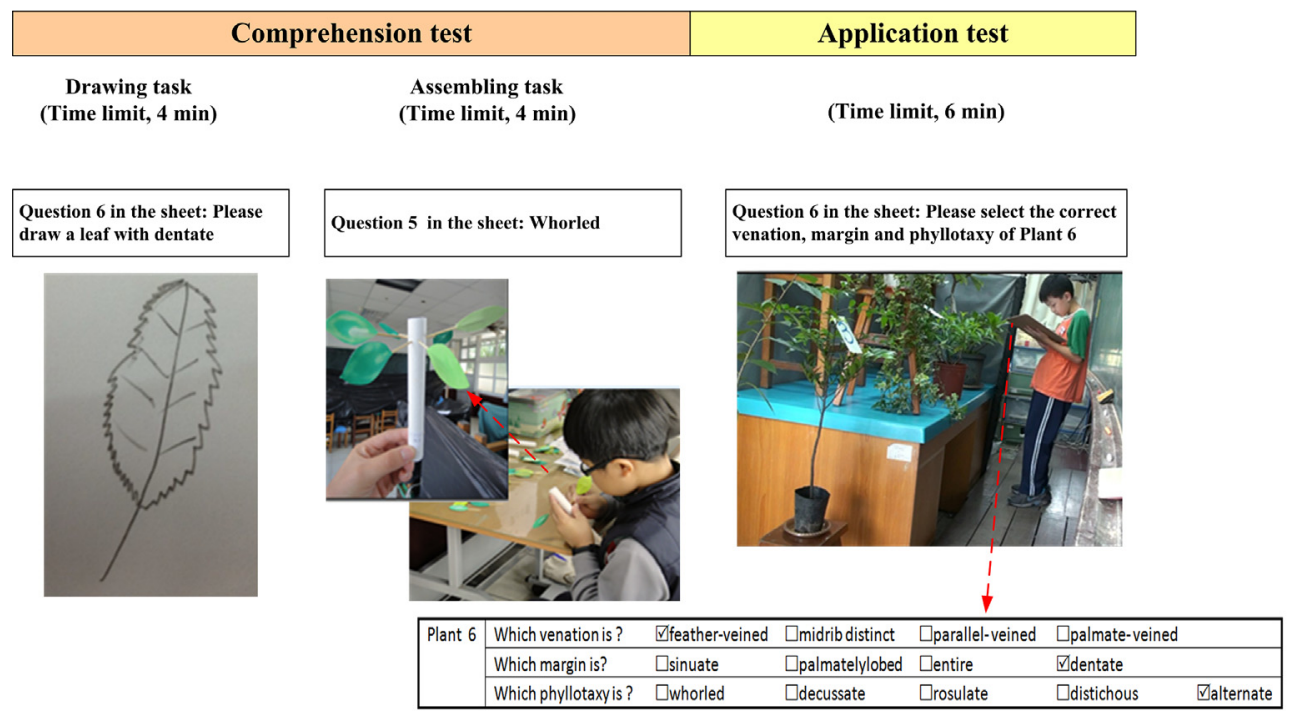

Fig. 3. Examples of comprehension and application test (note that the original materials were presented in the Chinese language).

In addition, as expected, high prior knowledge students needed less time to learn and achieved higher performance on both tests than low prior knowledge students.

The results of this experiment suggest that the redundancy effect was found for the low prior knowledge students. The photo of the plant on the mobile device and by the real plant in the physical environment were treated by the low prior knowledge students as information sources that are intelligible in isolation, with one of them being redundant (e.g., Cerpa et al., 1996; Kalyuga et al., 1999; Mayer \& Johnson, 2008; Rasch \& Schnotz, 2009). Possibly, they were not able to see the complementary value of the 3D information of the real plant to the 2D information in the photo of the plant since they were not able to distinguish between essential and non-essential elements of the representations (e.g., Moreno et al., 2011).

In Experiment 2 we investigated whether the redundancy effect could be avoided for low prior knowledge students by replacing the photos of plants on the mobile device by schematic hand drawings of plants. Hand drawings differ from photos in the sense that they can be used to depict only the important aspects of the plant leaves in a schematic representational format, and consequently, can be used as cue to focus the learner's attention on the relevant aspects of the leaves in the real plant. It can be assumed that this feature will stimulate low prior knowledge students to consider the hand drawings and the real plants as unintelligible, thereby creating a positive complementary effect of both representations. In Experiment 2 it was assumed that by using hand drawings of plants and real plants in the mobile device supported learning environment, redundancy effects could be avoided, especially for students with low prior knowledge. Consequently, they were expected to be better able to identify the important features in the real plants, which would lead to better learning in the condition with texts, hand drawings and real plants than in the condition with texts, photos and real plants.

\section{Experiment 2}

Experiment 2 compared the condition with texts, photos and authentic objects from Experiment 1 to a condition in which the photos were replaced by hand drawings. Similar to Experiment 1, the effects of the different compositions of media were studied as a function of prior knowledge, with low and high prior knowledge students participating in each condition.

It was hypothesized that an interaction would be found between composition of representations and prior knowledge (Hypothesis 3 ). More specifically, low prior knowledge students in the text with hand drawing and authentic objects condition were expected to spend less time and achieve higher test performance than the low prior knowledge students in the text with photos and real plants condition resulted from a redundancy effect (Hypothesis 3a), whereas, high prior knowledge students were expected to profit from both compositions of media to the same extent (Hypothesis 3b). In addition, it was expected that high prior knowledge students would spend less learning time and achieve higher test performance than low prior knowledge students in both conditions (Hypothesis 4).

Table 1

Means and SDs of the dependent variables.

\begin{tabular}{|c|c|c|c|c|c|c|c|c|}
\hline \multirow[t]{3}{*}{ Measure } & \multicolumn{4}{|l|}{$\mathrm{TP}$} & \multicolumn{4}{|l|}{ ТРО } \\
\hline & \multicolumn{2}{|c|}{ High prior knowledge } & \multicolumn{2}{|c|}{ Low prior knowledge } & \multicolumn{2}{|c|}{ High prior knowledge } & \multicolumn{2}{|c|}{ Low prior knowledge } \\
\hline & $M$ & $S D$ & $M$ & $S D$ & $M$ & $S D$ & $M$ & $S D$ \\
\hline Learning time (min) & 4.88 & 1.34 & 5.97 & 1.06 & 5.05 & 0.96 & 6.23 & 1.71 \\
\hline $\begin{array}{l}\text { Comprehension test } \\
\text { performance }(0-13)\end{array}$ & 9.60 & 1.84 & 8.00 & 1.36 & 8.73 & 2.94 & 4.73 & 2.25 \\
\hline $\begin{array}{l}\text { Application test } \\
\quad \text { performance }(0-18)\end{array}$ & 13.20 & 1.70 & 9.67 & 2.50 & 12.27 & 2.31 & 7.73 & 2.25 \\
\hline
\end{tabular}




\subsection{Method}

\subsubsection{Participants}

Eighty-one fifth-grade students (Mean age was around 11 years; 45 boys and 36 girls) from a primary school in Taiwan participated in this study. None of these students participated in the first experiment. The same teacher had taught all participants the essential concepts about leaf morphology of plants 18 months ago. Identical to Experiment 1, the participants' scores on a prior knowledge test were used to divide the participants across the high and low prior knowledge groups. The lowest score for the test was 0 points and the highest score was 10 points. Six points was used as a cutoff point and the 14 participants who got 6 points were not invited to participate in the formal experiment. There were 35 participants (about 43\% of participants), who got a pre-test score higher than 6 points and 32 participants (about $40 \%$ of participants), who got the pre-test score lower than 6 points. The 32 participants who got a pre-test score lower than 6 points were selected as low prior knowledge group $(M=4.44, S D=1.08)$. To create equal numbers for the experimental conditions, 32 participants that were randomly selected from the participants who got a pre-test score higher than 6 points were identified as the high prior knowledge group $(M=7.78, S D=0.79)$.

\subsubsection{Design}

A 2 (composition of representations: text-picture-and object vs. text-hand drawing-and object) $\times 2$ (prior knowledge: high vs. low) between-subjects design was used to address the hypotheses in this study. The resulting four conditions were high prior knowledge students learning with text-picture-and object, high prior knowledge students learning with text-hand drawing-and object, low prior knowledge students learning with text-picture-and object, and low prior knowledge students learning with text-hand drawing-and object. Participants were randomly assigned to the four conditions in such a way that each condition contained 16 participants.

\subsubsection{Materials}

Learning materials for introducing the basic knowledge of leaf features were the same as what were used in Experiment 1.

Learning materials for introducing the three features of leaves including two versions (TPO and THO) that were revised from the learning materials used in the studies of Liu et al. (2012) with the help of the same primary school teacher that was involved in Experiment 1 . The TPO condition in this experiment was identical to the TPO condition of Experiment 1. For the THO condition, the photos of plants used in the TPO condition were replaced by hand drawings of the plants that were made by the research team. Fig. 2 visualizes the differences between the TPO and THO conditions using the fourth screen of the experiment as an example. This screen presented the green maple plant to introduce the decussate phyllotaxy. Note that in all conditions, texts, photos and hand drawings were presented on a mobile device (i.e., tablet pc) and the real plant was available in the physical environment.

\subsubsection{Apparatus procedure}

The apparatus, measures and procedures were identical to those used in Experiment 1.

\subsubsection{Results and discussion}

The data of the learning phase were analyzed with 2 (composition of representations: THO vs. TPO) $\times 2$ (prior knowledge: High vs. Low) univariate analyses of variance (ANOVA) with learning time as dependent variable, and multivariate analyses of variance (MANOVA) with comprehension and application test scores as dependent variables. Statistical significance for all tests was set at $p<0.05$. The data of the learning and test phases are presented in Table 2.

3.1.5.1. Learning time. For the dependent variable learning time, the ANOVA results revealed neither main effects of composition of representations, $F(1,60)<1$, ns and prior knowledge, $F(1,60)<1$, ns, nor an interaction between both factors, $F(1,60)<1, n s$.

3.1.5.2. Comprehension and application performance. The results of 2 (composition of representations) $\times 2$ (prior knowledge) MANOVA with comprehension and application performance as dependent variables revealed a significant main effect of the composition of representations, Wilks's $\lambda=0.84, F(2,59)=5.66, p<0.01, \eta_{p}^{2}=0.16$, with participants in the THO condition outperforming participants in the TPO condition. In addition, a significant main effect of prior knowledge was found, Wilks's $\lambda=0.77, F(2,59)=8.91, p<0.001, \eta_{p}^{2}=0.23$, indicating that participants with high prior knowledge performed significantly better than participants with low prior knowledge. The interaction between composition of representations and prior knowledge was not significant, Wilks's $\lambda=0.99 ; F(2,59)<1, n s$.

The univariate ANOVAs that were computed with the Bonferroni correction set at alpha level $<0.05 / 2=0.025$, revealed the following results. With regard to comprehension performance, the main effect of composition of representations was significant, $F(1,60)=8.34$, MSE $=5.07, p<0.01, \eta_{p}^{2}=0.12$, with participants in the THO condition outperforming participants in the TPO condition. The main effect of prior knowledge was also significant, $F(1,60)=16.88$, MSE $=5.07, p<0.001, \eta_{p}^{2}=0.22$, indicating that participants with high prior

Table 2

Means and SDs of the dependent variables.

\begin{tabular}{|c|c|c|c|c|c|c|c|c|}
\hline \multirow[t]{3}{*}{ Measure } & \multicolumn{4}{|l|}{ TPO } & \multicolumn{4}{|l|}{ THO } \\
\hline & \multicolumn{2}{|c|}{ High prior knowledge } & \multicolumn{2}{|c|}{ Low prior knowledge } & \multicolumn{2}{|c|}{ High prior knowledge } & \multicolumn{2}{|c|}{ Low prior knowledge } \\
\hline & $M$ & $S D$ & $M$ & $S D$ & $M$ & $S D$ & $M$ & $S D$ \\
\hline Learning time (min) & 6.44 & 0.93 & 6.24 & 1.04 & 6.04 & 1.68 & 6.26 & 0.96 \\
\hline $\begin{array}{l}\text { Comprehension test } \\
\text { performance }(0-13)\end{array}$ & 8.13 & 1.54 & 5.31 & 2.80 & 9.25 & 2.11 & 7.44 & 2.37 \\
\hline $\begin{array}{l}\text { Application test } \\
\text { performance }(0-18)\end{array}$ & 10.63 & 1.89 & 9.06 & 1.88 & 11.69 & 1.74 & 10.69 & 2.18 \\
\hline
\end{tabular}


knowledge performed significantly better than participants with low prior knowledge. The interaction between composition of representations and prior knowledge failed to reach significance, $F(1,60)<1, n s$.

For application performance, the main effect of composition of representations was significant, $F(1,60)=7.75, \mathrm{MSE}=3.73, p<0.01$, $\eta_{2}^{p}=0.11$, with participants in the THO condition performing better than the participants in the TPO condition. The main effect of prior knowledge was also significant, $F(1,60)=7.05$, MSE $=3.73, p<0.025, \eta_{p}^{2}=0.11$, indicating that participants with high prior knowledge performed significantly better than participants with low prior knowledge. The interaction between composition of representations and prior knowledge failed to reach statistical significance, $F(1,60)<1, n s$.

In summary, both low and high prior knowledge students achieved better with the combination of hand drawings of plants on the mobile device and real plants in the physical environment than with the combination of photos of plants on the mobile device and real plants in the physical environment, as indicated by performance on the comprehension and application tests. The results could echo to the findings of Dwyer 1967 (cited in Dwyer, 1972) that realistic photographs are a less effective visual illustration format for complementing verbal representations than simple line drawings. As expected, and as a replication of Experiment 1, the results suggest that low prior knowledge students were not able to recognize the differences between the photos of plants and the real plants and, consequently, suffered from a redundancy effect. Instead, they could use the hand drawings to support them in identifying the important features of real plants, which made this combination of representations complementary for them. For high prior knowledge students the combination of hand drawings and real plants was also better than the combination of photos and real plants, indicating that it was easier for them too to use hand drawings instead of photos to identify the important features of real plants. Although, comprehension and application test performance of the high prior knowledge students was generally higher than that of the low prior knowledge students, this difference did not differ as a function of the composition of representations.

An alternative explanation for the higher learning performance in the THO condition than in the TPO condition is based on the variability effect (Paas \& Van Merriënboer, 1994; Renkl, Stark, Gruber, \& Mandl, 1998). The variability effect, which was introduced by Paas and Van Merriënboer (1994), holds that exposure to a highly varied sequence of problems and solutions to those problems imposes a higher cognitive load, but helps learners determine the range of applicability of constructed schemata. For the present study it could be argued that the combination of hand drawings of plants on the mobile device and real plants in the physical environment (THO) is more variable than the combination of photos of plants on the mobile device and real plants in the physical environment (TPO), and, according to the variability effect, might have resulted in the construction of a better schema and better test performance.

\section{General discussion}

Mobile device supported learning in physical environments has been widely used in recent years (Liu et al., 2009; Sung et al., 2010). However, there are some cognitive challenges in such learning environments that need to be dealt with before they can become effective learning environments. Building on the findings of Liu et al. (2012), this study examined the effects of compositions of multiple representations in the form of text, photos, schematic hand drawings, and real plants on learning of plant leaf morphology by primary school students.

Hypothesis (1a) of Experiment 1 that low prior knowledge students would achieve higher learning performance in a condition with text and photos of plants on a mobile device than in a condition with text and photos on a mobile device and real plants in the physical environment was confirmed. This result indicated that low prior knowledge students do not yet possess the appropriate cognitive schema to identify the differences between the information conveyed by photos of plants and real plants, and, therefore they are not able to exploit the complementary function of the different representations. Consequently, they focus on corresponding surface characteristics of the different representations (e.g., Cook et al., 2008), which makes the representations intelligible in isolation, with one of them being redundant. For high prior knowledge the same advantage for the TP condition over the TPO condition was found. However, this effect was explained in terms of the outcome of the sum of a positive complementary effect and a negative split-attention effect of the different representations. High prior knowledge students were expected to possess a cognitive schema that enables them to profit from the complementary function of the different representations (Ainsworth, 2006). However, this positive effect was negated by the requirement to mentally integrate the spatially separated representations on the mobile device and the real plants in the physical environment (i.e., split-attention effect). Experiment 2 compared the combination of text, photos and authentic objects that was used in Experiment 1 to a combination of text, hand drawings and authentic objects among low and high prior knowledge students. Hypothesis (3b) that especially low prior knowledge students would be able to learn more from the combination of text, hand drawing and real plant than from the combination of text, photo and real plant, could not be confirmed. Both the high and low prior students showed superior performance in the condition with hand drawings.

The results of both experiments suggest that the combination of photos of plants and real plants objects is redundant for low prior knowledge students, who do not have the necessary knowledge to recognize differences between the 2-dimensional information of the photos and the 3-dimensional information of the authentic objects. Consequently, using real plants in the physical environment with text and photos of plants on a mobile device, or vice versa, does not seem to make sense. By using schematic hand drawings instead of photos the differences with authentic objects become clearer, thereby enabling both low and high prior knowledge students to recognize differences and profit from the complementary information conveyed by both information sources. However, it is not clear whether such a condition with hand drawings is better than a condition with only text and photos of plants (TP condition of Exp. 1), because the latter conditions were not considered in Experiment 2. Future research could compare those conditions to find the most effective strategy.

In addition to finding the most effective strategy, it could be interesting for future research to investigate under what conditions the use of real plants is effective for learning. Anecdotal evidence from our previous studies (Liu et al., 2012, 2013) and this study suggests that students like to interact with real plants. The importance of "authentic objects" for learning was also mentioned in other studies. For example, students indicated that they like to learn about science through real-object observation in a context-aware ubiquitous learning environment (Hwang, Shi, \& Chu, 2011), and observational records indicated that students showed high motivation when the teacher presented a scientific demonstration with authentic object Lin, Liu, \& Chu, 2011). However, empirical evidence for the beneficial effects of using authentic objects for learning is lacking. In the context of the present results, it would be interesting to study whether presenting the 
real plants before presenting the text and photos would lead to better learning results. In addition, it would be interesting to design a learning condition with less split attention, for example, by instructing the students to physically integrate the different representations by holding the mobile device next to the plants.

In addition, future research could be directed at finding alternative ways to emphasize the structural differences between photos and authentic objects for low prior knowledge learners. Previous research of De Koning, Tabbers, Rikers, and Paas (2009, 2010) and Liu et al. (2013) suggests that using attentional cueing is a promising instructional technique to make those differences more salient to novices and improve their learning. It should be noted that the schematic hand drawings that were used in the present study can also be considered as a kind of cue.

Finally, according to the cognitive theory of multimedia learning (Mayer, 2005, 2009) a specific channel becomes overloaded when multiple learning sources must be processed by one channel (e, g., visual/pictorial channel) in a limited time. Future research could replace the texts embedded in the tablet PC with narration and examine the effects.

A limitation of the current study is that we only have indirect evidence for our claims regarding the cognitive processes underlying redundancy and split-attention effects of low and high prior knowledge students. Although previous research makes clear that the different cognitive schemas that novices and more advanced learners possess affect the way in which they deal with learning materials, our assumptions about how low and high prior knowledge students dealt with information conveyed by a photo of a plant, a schematic hand drawing of a plant, and the real plant could not be supported by the collected data. It is very important for future research to collect more direct evidence about the way in which the level of students' prior knowledge affects the way in which they deal with multiple representations, for example by using eye-tracking and verbal protocols.

Another limitation of this study is that cognitive load was not measured, which does not allow us to present strong empirical support for our arguments regarding cognitive load effects in general, the verification of the fact that the higher performance of high prior knowledge students was not obtained by and more specifically, regarding redundancy and split-attention effects for low and high prior knowledge learners. It would be especially interesting for future research to measure the different types of cognitive load that different compositions of multiple representations impose on low and high prior knowledge students (Leppink, Paas, Van der Vleuten, Van Gog, \& Van Merriënboer, in press). As an example, in terms of cognitive load theory, it can be assumed that the magnitude of intrinsic load (useful for learning) imposed on more advanced learners increases due to the structural comparisons they make between the photos and the real plants. At the same time, however, the comparison process between the spatially separated photos and real plants imposes an extraneous load (not useful for learning) due to a split-attention effect. Because the extraneous load is unavoidable, its magnitude determines how much of the limited working memory resources remain for the more sophisticated processing in terms of structural comparisons. The newly developed instrument of Leppink et al. (in press) could provide more insight into these and similar assumptions related to the overall and specific types of cognitive load.

Finally, the use of dichotomization of participants regarding their prior knowledge, and the use of non-parallel pretest and posttest could be considered as methodological limitations of this study. With regard to the first methodological limitation, the use of dichotomization has been criticized by researchers (e.g., Humphreys, 1978; Humphreys \& Fleishman, 1974; Vargha, Rudas, Delaney, \& Maxwell, 1996). However, DeCoster, Iselin, and Gallucci (2009) indicated that in certain cases (such as the extreme group design used in this study), dichotomization is just as effective as and even more effective than considering the continuous variable. According to the opponents, extreme group designs would misrepresent the relations among variables found in the real world, give the illusion of experimental control to designs that lack it, and reduce the size of the observed relations (Humphreys, 1978; Humphreys \& Fleishman, 1974) and reduce the power of statistical tests and incorrectly leading to significant results (e.g., Vargha et al., 1996). Future studies on the same issue should use prior knowledge as a continuous variable in the experimental design. The second methodological limitation regarding the non-identical pretest and posttest holds that there is a possibility of assignment bias and the posttest scores might have been obscured. However, we carefully randomized the experimental conditions, and all participants had the same educational background. Most importantly, we found clear performance differences in the expected direction on the posttest between the students who had been categorized as low and high prior knowledge students by the pretest. Although, it is unlikely that the posttest scores were not reflecting real knowledge gains, future research should use a pretest and a posttest with identical questions.

The results of this study also have important practical implications. Mobile device supported learning environments are increasingly used in today's educational systems (Huang, Chiu, Liu, \& Chen, 2011; Liu, 2007). This study shows that it is not effective to combine information on a mobile device with information in the physical environment. Particularly, it is important to realize that it might be too difficult for students to mentally integrate different representations, such as photos of authentic objects and the authentic objects. In the case that authentic objects have to be used in a learning environment, it is better to combine them with schematic hand drawings than with photos.

\section{Acknowledgements}

The authors would like to thank the assistance of Chia-Ling Kao and Chen-Yi Wang in this study. We would also like to thank the administration, the teachers and the students of Taoyuan Hsin Yi elementary school who participated in this study. Furthermore, we would like to thank the kind assistance and helpful comments of the editor of Computers \& Education and the anonymous reviewers of this paper. Finally, we would like to thank the National Science Council of the Republic of China, Taiwan, for financially supporting this research under Contract No. 98-2628-S-008-001-MY3, NSC 100-2628-S-008-001-MY3, and NSC 101-2511-S-008-004-MY3.

\section{References}

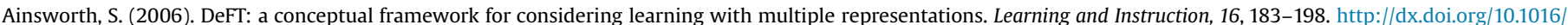
j.learninstruc.2006.03.001.

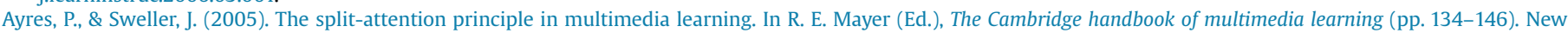
York: Cambridge University Press. 


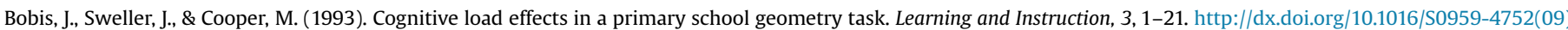
80002-9.

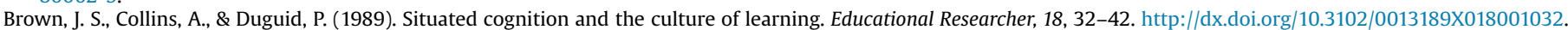

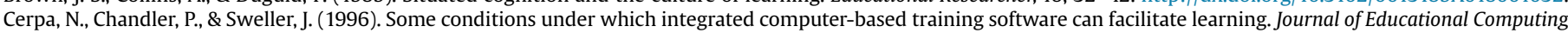
Research, 15, 345-367. http://dx.doi.org/10.2190/MG7X-4J8N-CKYR-P06T.

Chandler, P., \& Sweller, J. (1991). Cognitive load theory and the format of instruction. Cognition and Instruction, 8, 293-332. http://dx.doi.org/10.1207/s1532690xci0804_2.

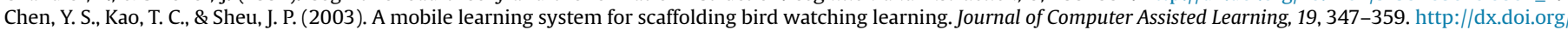
10.1046/j.0266-4909.2003.00036.x.

Chi, M. T. H., Glaser, R., \& Farr, M. J. (1988). The nature of expertise. Hillsdale, NJ: Lawrence Erlbaum Associates.

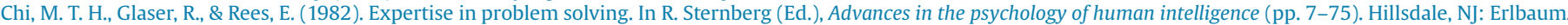

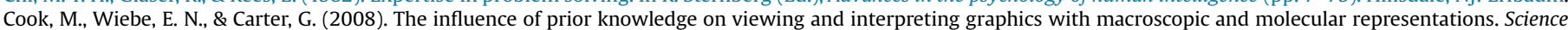
Education, 92, 848-867. http://dx.doi.org/10.1002/sce.20262.

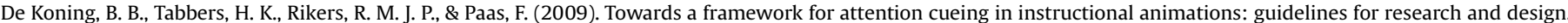
Educational Psychology Review, 21, 113-140. http://dx.doi.org/10.1007/s10648-009-9098-7.

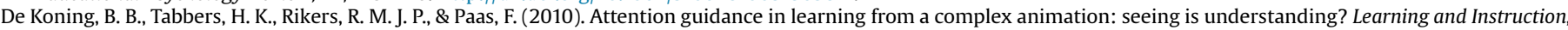
20, 111-122. http://dx.doi.org/10.1016/j.learninstruc.2009.02.010.

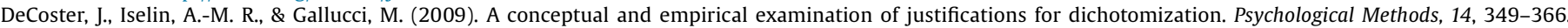
http://dx.doi.org/10.1037/a0016956.

Dwyer, F. M. (1972). A guide for improving visualized instruction. State College, PA: Learning Services.

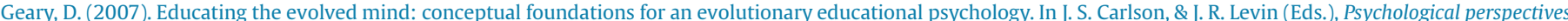
on contemporary educational issues (pp. 1-99). Greenwich, CT: Information Age Publishing.

Geary, D. (2008). An evolutionarily informed education science. Educational Psychologist, 43, 179-195. http://dx.doi.org/10.1080/00461520802392133.

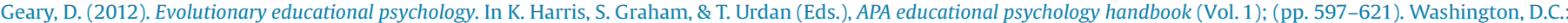
American Psychological Association.

Heinich, R., Molenda, M., Russell, J. D., \& Smaldino, S. (2001). Instructional media and technologies for learning (7th ed.). Columbus: Merrill/Prentice Hall.

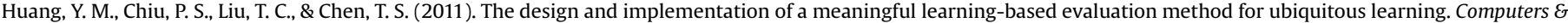
Education, 57, 2291-2302. http://dx.doi.org/10.1016/j.compedu.2011.05.023.

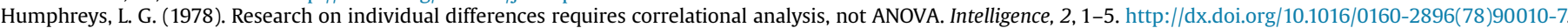

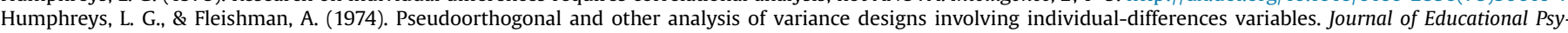
chology, 66, 464-472. http://dx.doi.org/10.1037/h0036539.

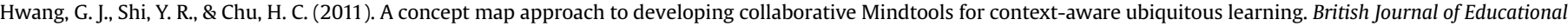
Technology, 42, 778-789. http://dx.doi.org/10.1111/j.1467-8535.2010.01102.x.

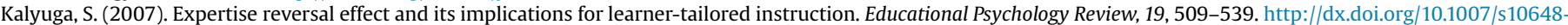
007-9054-3.

Kalyuga, S., Ayres, P., Chandler, P., \& Sweller, J. (2003). The expertise reversal effect. Educational Psychologist, 38, $23-31$.

Kalyuga, S., Chandler, P., \& Sweller, J. (1998). Levels of expertise and instructional design. Human Factors, 40, 1-17. http://dx.doi.org/10.1518/001872098779480587.

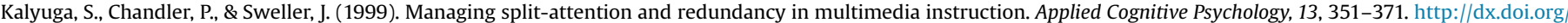
10.1002/(SICI)1099-0720(199908)13:4<351::AID-ACP589>3.0.CO;2-6.

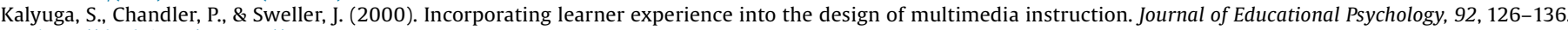
http://dx.doi.org/10.1037//0022-0663.92.1.126.

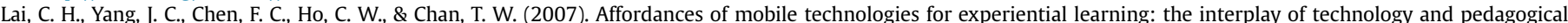
practices. Journal of Computer Assisted Learning, 23, 326-337. http://dx.doi.org/10.1111/j.1365-2729.2007.00237.x.

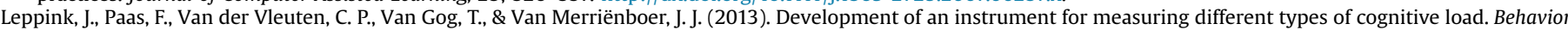
Research Methods, 45, 1058-1072. http://dx.doi.org/10.3758/s13428-013-0334-1.

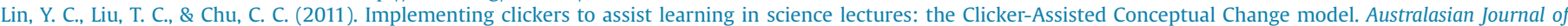
Educational Technology, 27, 979-996.

Liu, T. C. (2007). Teaching in a wireless learning environment: a case study. Educational Technology E Society, 10, 107-123.

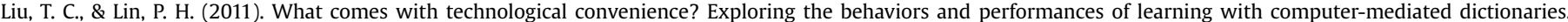
Computers in Human Behavior, 27, 173-183. http://dx.doi.org/10.1016/j.chb.2010.08.016.

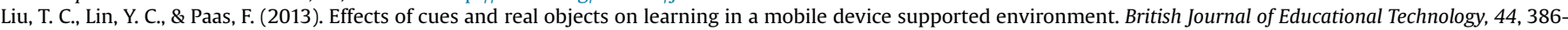
399. http://dx.doi.org/10.1111/j.1467-8535.2012.01331.x

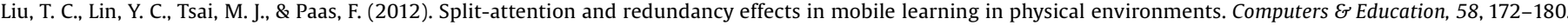
http://dx.doi.org/10.1016/j.compedu.2011.08.007.

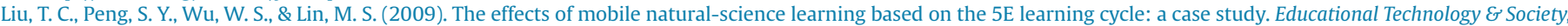
12, 344-358.

Mayer, R. E. (2005). The Cambridge handbook of multimedia learning. Cambridge: Cambridge University Press.

Mayer, R. E. (2009). Multimedia learning (2nd ed.). New York: Cambridge University Press.

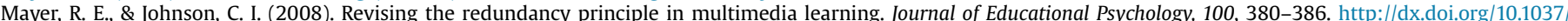
0022-0663.100.2.380.

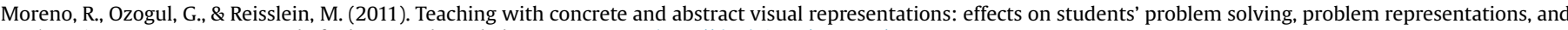
learning perceptions. Journal of Educational Psychology, 103, 32-47. http://dx.doi.org/10.1037/a0021995.

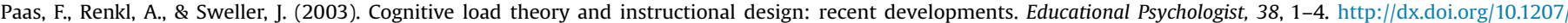
S15326985EP3801_1.

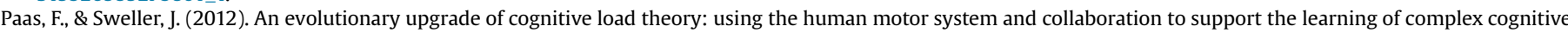
tasks. Educational Psychology Review, 24, 27-45. http://dx.doi.org/10.1007/s10648-011-9179-2.

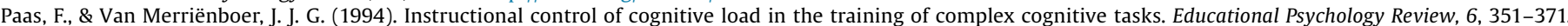
http://dx.doi.org/10.1007/BF02213420.

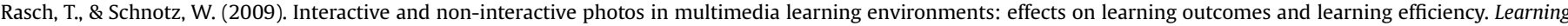
and Instruction, 19, 411-422. http://dx.doi.org/10.1016/j.learninstruc.2009.02.008.

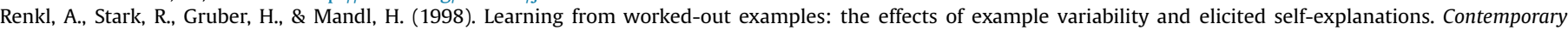
Educational Psychology, 23, 90-108. http://dx.doi.org/10.1006/ceps.1997.0959.

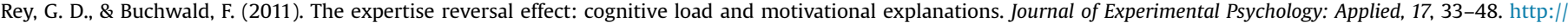
dx.doi.org/10.1037/a0022243.

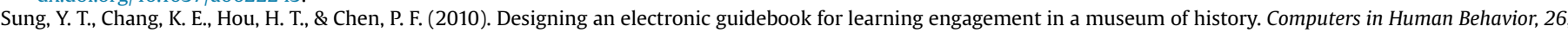
74-83. http://dx.doi.org/10.1016/j.chb.2009.08.004.

Sweller, J., Ayres, P. \& Kalyuga, S. (2011). Cognitive load theory, Berlin: Springer.

Sweller, J., Van Merriënboer, J. J. G., \& Paas, F. (1998). Cognitive architecture and instructional design. Educational Psychology Review, 10, 251-296.

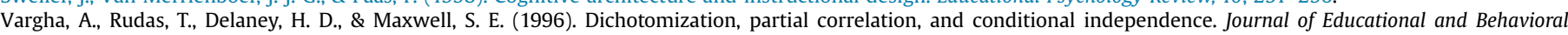
Statistics, 21, 264-282. http://dx.doi.org/10.2307/1165272. 